\title{
Supergalactic Structure of Energy-Angle Correlations
}

\author{
J.P. Lundquist ${ }^{* 1,2 \dagger}$ and P. Sokolsky ${ }^{3}$ for the Telescope Array Collaboration \\ 1 Dept. of Physics, Sungkyunkwan University, Suwon 440-746, Republic of Korea \\ 2 Inst. for Phys. Sci. and Tech., University of Maryland, College Park, MD 20742, USA \\ 3 High Energy Astrophysics Inst. and Dept. of Physics and Astronomy, University of Utah, Salt \\ Lake City, UT, USA
}

\begin{abstract}
Evidence for the supergalactic structure of multiplets (energy-angle correlations) has previously been shown using ultra-high energy cosmic ray (UHECR) data from Telescope Array (TA) with energies above $10^{19} \mathrm{eV}$. The supergalactic deflection hypothesis (that UHECR sources and intervening magnetic fields are correlated) is measured by the all-sky behavior of the strength of intermediate-scale correlations. The multiplets are measured in spherical surface wedge bins of the field-of-view to account for uniform and random magnetic fields. The structure found is consistent with the previously published energy spectrum anisotropy results of TA and toy-model simulations of a supergalactic magnetic sheet. The 7 year data post-trial significance of this feature appearing by chance, on an isotropic sky, was found by Monte Carlo simulation to be $\sim 4 \sigma$. The analysis has now been applied to 10 years of data.
\end{abstract}

36th International Cosmic Ray Conference -ICRC2019-

July 24th - August 1st, 2019

Madison, WI, U.S.A.

\footnotetext{
*Speaker.

†jplund@umd.edu

${ }^{\ddagger}$ for collaboration list see PoS(ICRC2019)1177
} 


\section{Introduction}

Large scale magnetic fields have been measured between clusters of galaxies which make up the supergalactic plane (the average matter distribution largely within the GZK cutoff of $100 \mathrm{Mpc}$ ) such as the Coma Cluster and a 3 Mpc field between Abell 0399 and Abell 0401 ([1], [2], [3]). It has also been shown that $\sim 90 \%$ of the universe's baryons are contained between galaxies ([4]), and that these may support formation of even larger intra-galactic scale magnetic fields [5].

Large scale magnetic fields suggest that energy dependent deflection of ultra-high energy cosmic rays (UHECR) may appear correlated with the SGP. Previous energy-position correlation (multiplet) searches for small scale magnetic deflections have not had significant results ([6],[7],[8]). This analysis uses intermediate-scale energy-position correlations to look for significant large scale magnetic structure. We report an update using ten years of Telescope Array (TA) data from the seven year result reported in [9].

\section{Data Set}

Surface detector (SD) data from May 11 of 2008 to 2019 is used for this analysis. The 2016 data is removed due to SD communication tower issues that caused a very high day to day variance, in the trigger delay variance each day in the year. This introduced non-physical anisotropies that are non-trivial to compensate for. Reconstructed event energy is determined by the SD array and renormalized by $1 / 1.27$ to match the calorimetric fluorescence detector energy scale [10]. The event reconstruction used is that of the "Hotspot" analysis [11]. The use of lower energies down to $10^{19.0} \mathrm{eV}$ requires tighter data cuts for good zenith angle and energy resolution.

Events in the data set match the following criteria: $\mathrm{E} \geq 10^{19.0} \mathrm{eV}$ (where detection efficiency is $\sim 100 \%$ ), at least four SDs triggered, arrival zenith angle $<55^{\circ}$, pointing direction error $<5^{\circ}$, shower core $>1.2 \mathrm{~km}$ from array boundary, shower lateral distribution fit $\chi^{2} /$ dof $<10$. After cuts, there were 3027 events in the seven year data set and there are 4321 using ten years of data.

The additional cuts on pointing direction error and boundary distance improve the zenith angle distribution agreement with the geometrical zenith exposure $g(\theta)=\sin (\theta) \cos (\theta)$. The azimuthal angle distribution agrees with a flat distribution. The energy spectrum is also in good agreement with the published spectrum [10].

Energy resolution and zenith angle resolution of events range from $\sim 10$ to $15 \%$ and $\sim 1.0^{\circ}$ to $1.5^{\circ}$, respectively, depending on core distance from the array boundary and improve with increasing energy. These resolutions are sufficient to search for UHECR energy anisotropies.

\section{Energy-Angle Correlations}

It's assumed that UHECR travel through uniform fields, Equation 3.1a, and random fields where the deflection variance is shown in Equation 3.1b ( $Z$ is mass number, $S$ distance, $B$ field strength, $E$ particle energy, and $L_{c}$ is mean magnetic field coherence length). The result is lower energy cosmic ray events are deflected to larger angles from their source than higher energy events [12]. Figure 1 shows a diagram of this energy dependent drift-diffusion. 


$$
\delta \approx 0.5^{\circ} \mathrm{Z} \frac{S B}{\mathrm{kpc} \mu \mathrm{G}} \frac{10^{20} \mathrm{eV}}{E} \quad \delta_{r m s} \approx 0.1^{\circ} Z \frac{B_{r m s}}{\mu \mathrm{G}} \frac{10^{20} \mathrm{eV}}{E} \sqrt{\frac{S L_{c}}{\mathrm{kpcpc}}}
$$

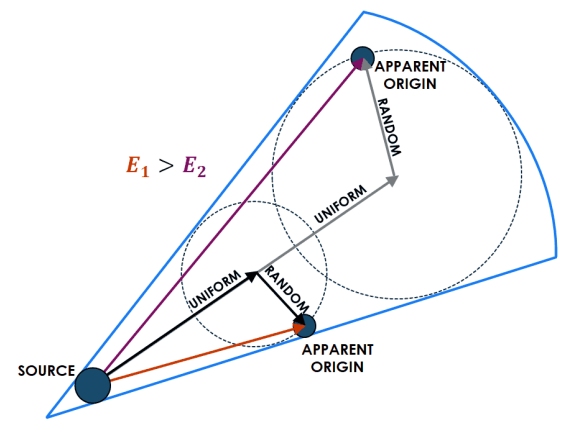

(a)

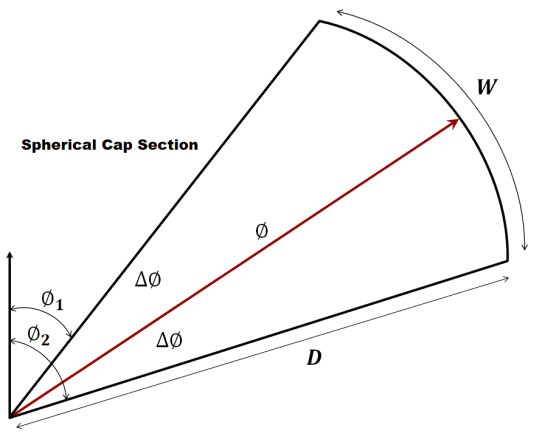

(b)

Figure 1: UHECR Drift-diffusion deflection "wedge" bins. (a) Two events having traveled through uniform and random magnetic fields. The purple vector represents the low energy event spherical arc and the red vector is a higher energy event. Uniform and random magnetic field components describe the average perpendicular to the field of view (FOV) sphere. Possible random field deflections are represented by dashed circles. (b) A spherical cap section, "wedge," is a simple shape that best encompasses the likeliest positions. Pointing direction is the spherical $\operatorname{arc} \phi$, width is $\Delta \phi$, and $D$ is the maximum angular distance.

\subsection{Correlation}

Correlations between energy and great circle angular distance of events are found using a ranked correlation, Kendall's $\tau_{b}$, that measures the strength of monotonic dependence [13]. Ranked correlation is the linear correlation between the separate ordering of the two variables of interest (Variable $x$ : 1st, 2nd, 3rd, etc. versus Variable $y$ : 5th, 1st, 4th, etc.). If $\tau_{b}=-1$ an increase (decrease) of $x$ always follows a decrease (increase) in $y$. For +1 an increase (decrease) of $x$ always follows an increase (decrease) of $y$. This removes magnetic model and composition assumptions and also detector exposure effects on the correlation strength.

The significance of a correlation (probability of $\tau_{b}=0$ ) is a function of correlation strength and sample size. This is found by permutations of the sample or the large sample limit that follows the standard normal distribution. Further details can be found in [9] and [13].

\subsection{Correlation Binning}

Possible UHECR deflections from sources were found by a scanned maximization of the significance of energy-angle correlations inside spherical cap sections, or "wedges," using seven years of TA data [9]. An example is shown in Figure 2(a). This was done at each point on an equal $2^{\circ}$ spaced grid on the FOV. Wedge bins are defined by a maximum angle radius, $\delta_{j}$, from grid point, $i$, and the boundaries of two azimuths. The angular distance between the wedge pointing direction, $\phi_{i}$, and an events azimuth is $\Delta \phi_{i j}$.

This bin shape requires a scan of four parameters at every grid point to maximize the significance. Though negative correlations are physically expected from magnetic deflections; the 
correlation sign, and strength, are not scanned for nor restricted. Reasonable deflection scenarios are taken into account by a large parameter space and are the following:

1. Energy Threshold, $E_{i}: 10$ to $100 \mathrm{EeV}, 5 \mathrm{EeV}$ steps.

2. Wedge Angular Distance, $D_{i}=\max \left(\delta_{i j}\right): 15^{\circ}$ to $90^{\circ}, 5^{\circ}$ steps.

3. Wedge Direction, $\phi_{i}: 0^{\circ}$ to $355^{\circ}, 5^{\circ}$ steps.

4. Wedge Width, $W_{i}=2 \max \left(\left|\Delta \phi_{i j}\right|\right): 10^{\circ}$ to $90^{\circ}, 10^{\circ}$ steps $\left(5^{\circ}\right.$ on each side of $\left.\phi_{i}\right)$.

Events are in the wedge if $E_{j} \geq E_{i} \& \delta_{i j} \leq D_{i} \&-W_{i} / 2 \leq \Delta \phi_{i j} \leq W_{i} / 2$ ( $i$ is the grid point). The parameters $\left(E_{i}, D_{i}, \phi_{i}\right.$, and $\left.W_{i}\right)$ are chosen for the minimum p-value of the energy-angle correlation, $\tau_{b}\left(\delta_{i j}, E_{j}\right)$ (maximum significance).

\subsection{Most Significant Correlation}

These parameters were scanned for seven years of TA data as shown in [9]. No parameters were changed for the ten year data update shown here. The most significant single correlation in 10 years of data is at $30.3^{\circ} \mathrm{SGB},-3.2^{\circ} \mathrm{SGL}, 11^{\circ}$ from M82 pointing towards the TA Hotspot, and shown in Figure 2(a). With 75 events $(\mathrm{E} \geq 35 \mathrm{EeV}) \tau_{b}=-0.412$ has pre-trial significance of $5.10 \sigma$. This is an increase from $4.58 \sigma$ at this grid point with 7 years of data using the same wedge and energy threshold parameters. Figure 2(b) shows a scatter plot of energy versus angular distance and a linear fit (Equation 3.1a with $Z=1$ ) results in an estimate of $S \times B=41.1 \mathrm{kpc} \times \mu \mathrm{G}$. If the source is assumed to be at the distance to M82 $(3.7 \mathrm{Mpc})$ the average uniform magnetic field required to cause this deflection would be $B=12 \mathrm{nG}$.

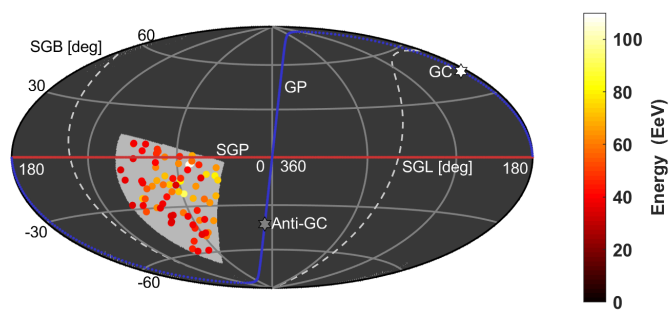

(a)

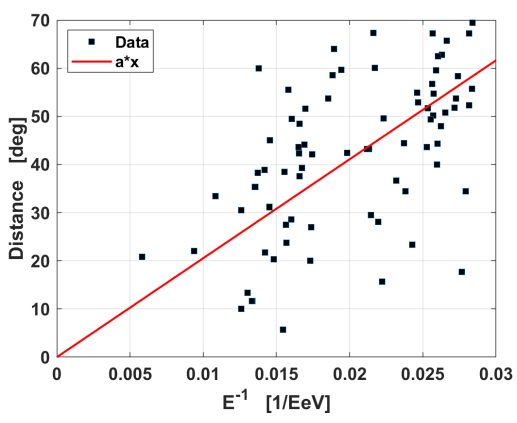

(b)

Figure 2: (a) Supergalactic Hammer-Aitoff projection of the 10 year maximum significance "wedge" at $30.3^{\circ} \mathrm{SGB},-3.2^{\circ} \mathrm{SGL}$. The correlation $\tau_{b}=-0.412$ with 75 data events has a pre-trial one-sided significance of $5.10 \sigma$. This increased from $4.58 \sigma$ at this grid point with 7 years of data. The energy threshold is $E_{i} \geq 35 \mathrm{EeV}$, wedge width $W_{i}=90^{\circ}$, angular distance $D_{i}=70^{\circ}$, and direction $\phi_{i}=120^{\circ}$. (b) Scatter plot of $1 / E_{j}$ versus angular distance $\delta_{j}$ in the wedge.

The post-trial significance of this particular correlation is not expected to be large, as the wedge scan parameter space is large. An individual is not the test of supergalactic structure.

\section{Simulations}

The analysis was applied to isotropic simulations for the seven year anisotropy significance calculation as described further in Section 5. A second simulation is a simple simulation of a 
supergalactic magnetic sheet resulting in an energy dependent diffusion of events away from the supergalactic plane. This is used to motivate the test statistic that was used to test the hypothesis of supergalactic sources and magnetic fields.

\subsection{Isotropic Simulation}

Actual data coordinates were used for the isotropic simulations. New energies were assigned to data positions by interpolating a large set of $\mathrm{MC}$ energies reconstructed through a surface detector simulation thrown with the HiRes/TA spectrum ([14], [15]). The number of events in each isotropic $\mathrm{MC}$ event set were the same as data in each $5 \mathrm{EeV}$ bin. This simulated the expected data given the detector configuration and on-time with no energy anisotropies.

\subsection{Supergalactic Magnetic Sheet}

A simple toy-model supergalactic magnetic sheet uses event deflections in SGB, proportional to 1 / energy according to Equation 3.1a, for a fraction of events in the isotropic simulations. A simulation, with an $F=65.7 \%$ isotropic fraction (1988 out of 3027 events) and $S \times B=18.47 \mathrm{kpc} \times \mu \mathrm{G}$, is shown in Figure 3(a). This is only an anisotropy of energies, event positions are isotropic.

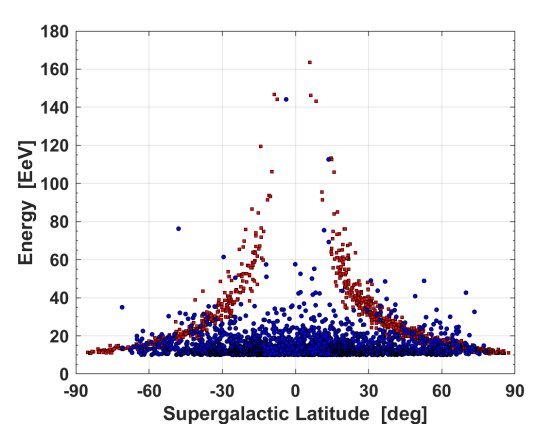

(a)

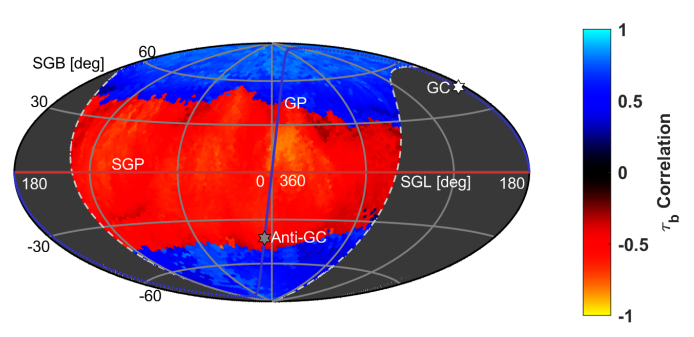

(b)

Figure 3: Toy-model supergalactic magnetic sheet simulation of anisotropic energies. (a) Blue circles are isotropic events $(F=65.7 \%)$. Red squares are anisotropic events magnetically diffused away from the supergalactic plane with $S \times B=18.47 \mathrm{kpc} \times \mu \mathrm{G}$. All event positions are isotropic and the energy spectrum is created according to the published TA results. (b) Projection of the energy-angle correlation strength $\tau_{b}$ inside wedges for all grid points. Solid curves indicate the galactic plane (GP) in blue and supergalactic plane (SGP) in red.

Unlike the isotropic simulations data coordinates are not used. On-time is simulated by sampling the trigger times of 264,499 data events with $\mathrm{E}>10^{17.7} \mathrm{eV}$. The azimuthal angle distribution is $0^{\circ}$ to $360^{\circ}$ uniform and the zenith angle distribution is $\mathrm{g}(\theta)=\sin (\theta) \cos (\theta)$ due to the flat $\mathrm{SD}$ array. Positions of energies are moved by $\delta_{k}$, for each event, $j$, in the anisotropic fraction assuming protons $(Z=1)$, and an $S \times B$. The energies, $E_{k}$, and their deflection from $S G B=0\left(\delta_{k}\right)$ are assigned to the closest isotropic $S G B_{j}$ value $\left(\min \left[\delta_{k}-S G B_{j}\right]\right)$ in random order. Further detail is in [9]

It can be seen via this simple model in the projection of the $\tau_{b}$, in Figure 3(b), that if there are magnetically induced energy-angle correlations clustered in the supergalactic plane, negative correlation wedges will be close to the supergalactic plane. Furthermore, since negative correlations 
viewed from the opposite direction appear as a positive correlations $\left(\left(x_{j}, y_{j}\right) \rightarrow\left(x_{j},-y_{j}\right)\right)$, positive correlations are expected at large distances from the supergalactic plane.

\section{Supergalactic Structure}

No single correlation tests the hypothesis that sources and magnetic fields are correlated with local large scale structure. And no single correlation can be significant when taking into account the $\sim 100,000$ scan parameter combinations at all 6553 grid points.

A test for supergalactic structure, of energy-angle correlations, is not necessarily a priori obvious. The mean $\left\langle\tau_{b}>\right.$ inside equal solid angle bins of angular distance $\left(S G B_{i}\right)$ from the supergalactic plane were used in the seven year data analysis. The single parameter chosen to test the supergalactic structure hypothesis was the curvature parameter, " $a$," of a parabolic fit $\left(a x^{2}+b x+c\right)$ to the $<\tau_{b}>$.

The curvature, "a", is simply the lowest order Taylor expansion term that can describe the symmetry around the SGP shown in simulation (Figure 3(b)). Greater correlation curvature means that the minimum negative correlation, and maximum positive correlation, averages are larger in magnitude. It also means that the minimum is closer to the supergalactic plane as shown in [9].

The large scale behavior of the correlation strength, $\tau_{b}$, was used because it is not explicitly scanned for and contains more information by its sign $( \pm)$ than the pre-trial significance. The pretrial significance of the correlations was not used so that the significance test was independent of the wedge scan for maximum energy-angle correlation significance.

The data significance of a supergalactic structure of energy-angle correlations the analysis was applied to the data, and the isotropic MC sets. The number of MC sets with a correlation curvature, $a$, greater than the data gives the probability that there is not a supergalactic structure of energy-angle correlations.

\section{Results}

The resulting data energy-angle correlations for seven years of data are shown in Figure 4(a). Individual correlations with the highest pre-trial significance are negative which means that there is a trend for the angular distance to increase with decreasing energy. This is the expectation for a grid point that happens to be near a UHECR source of magnetically scattered events. It can be seen that the negative $\tau_{b}$ correlations appear well correlated with the supergalactic plane.

Figure 4(b) shows the seven year data result of the mean $\tau_{b}$ correlation inside equal solid angle bins parallel to the supergalactic plane. The curvature was $a=2.4 \times 10^{-4}$ a minimum at -1.1 SGB. The data correlations have a very similar form to that of the supergalactic magnetic sheet simulation, shown in Figure 3(b), that has a slightly higher $a=2.5 \times 10^{-4}$ at $-1.7 \mathrm{SGB}$. By applying this analysis to isotropic MC sets and counting the number of MC with an $a$ parameter greater than data the post-trial significance of the supergalactic structure of multiplets was found to be $\sim 4 \sigma[9]$.

Figure 4(b) shows the 10 year mean $\tau_{b}$ correlation with no new scan for maximum correlation significances. The parabola curvature is $a=1.6 \times 10^{-4}$ and the minimum is at $1.2 \mathrm{SGB}$. It can be seen that the correlations are similar to the seven year result but the supergalactic structure may not be quite as significant. 


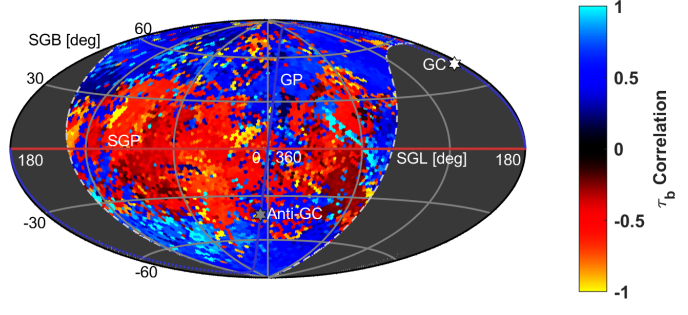

(a)

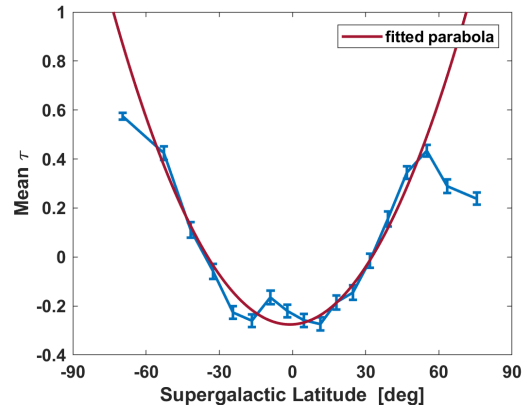

(b)

Figure 4: Seven year data result. (a) Projection of the correlation strength $\tau_{b}$ for all grid points. Negative correlations expected for magnetic deflections are apparent around the supergalactic plane. Solid curves indicate the galactic plane (GP) in blue and supergalactic plane (SGP) in red. White and grey hexagrams indicate the Galactic center (GC) and anti-galactic center (Anti-GC) respectively. (b) Mean $\tau_{b}$ inside equal solid angle bins. The correlation curvature is $a=2.4 \times 10^{-4}$.

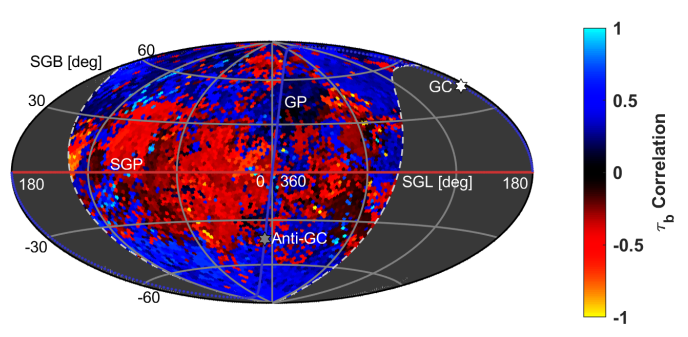

(a)

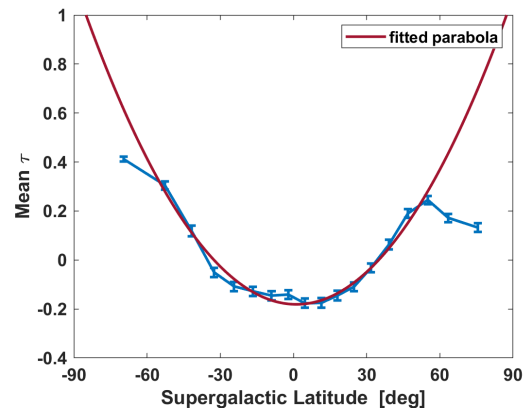

(b)

Figure 5: 10 year data result. (a) Projection of the correlation strength $\tau_{b}$ for all grid points. Negative correlations expected for magnetic deflections are apparent around the supergalactic plane. (b) Mean $\tau_{b}$ inside equal solid angle bins. The correlation curvature is $a=1.6 \times 10^{-4}$.

\subsection{Alternate Tests}

There are other apparent correlation symmetries around the supergalactic plane that could be used to test a new independent data set (TAx4 data, for example [16]). Inside equal solid angle bins of supergalactic latitude the number of negative correlations, the mean correlation $\operatorname{sign}\left(\tau_{b}\right) \times \sigma$, and the mean estimated uniform magnetic field are examples.

The mean correlation $\operatorname{sign}\left(\tau_{b}\right) \times \sigma$ is shown in Figure 6.1 and combines both values output by the $\tau_{b}$ correlation, the correlation sign and the scanned for (in the seven years of data) significance of the correlation. The curvature parameter for the seven year data set is $a=0.0013$ and the ten year data set is $a=0.0010$ which is less of a change between the two data sets than the mean $\tau_{b}$.

\section{Summary}

Intermediate-scale energy-angle correlations inside spherical cap sections, "wedges," were previously shown, using seven years of Telescope Array (TA) data, to have a $\sim 4 \sigma$ correlation with 


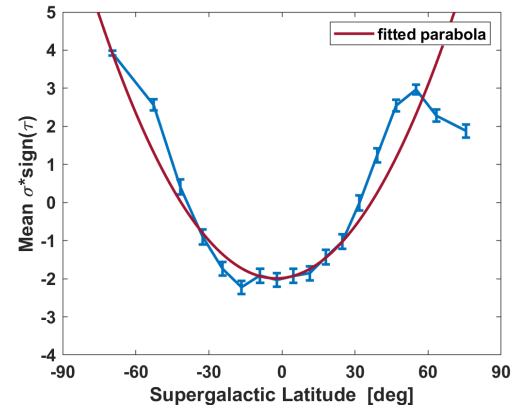

(a)

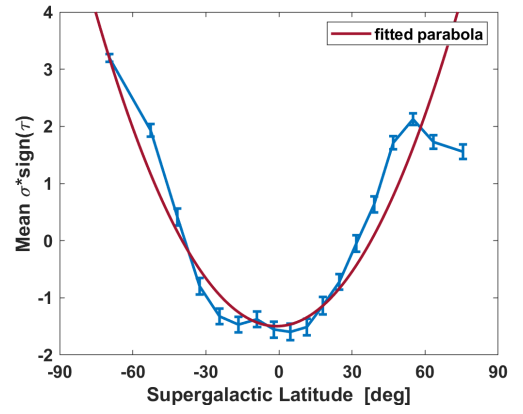

(b)

Figure 6: The mean $\operatorname{sign}\left(\tau_{b}\right) \times \sigma$ inside equal solid angle bins of SGL. (a) Seven years of data with a curvature parameter $a=0.0013$. (b) Ten years of data with a curvature parameter $a=0.0010$.

the supergalactic plane. Though the ten year data significance has not yet been updated this may be evidence of large scale magnetic diffusion of UHECR from sources correlated with local large scale structure. The highest significance single energy-angle correlation has increased from a pretrial $4.6 \sigma$ significance to pre-trial $5.1 \sigma$ with no new scan of wedge parameters. This correlations origin point is $11^{\circ}$ from M82 and lies over the TA Hotspot. Confirmation of this result may be done once sufficient data is collected by the TA expansion to TAx4 [16].

\section{References}

[1] de Vaucouleurs, G., Astrophys. J. 202 (1975) 610.

[2] Bonafede, A. et al., Astron. Astrophys. 513 (2010) A30.

[3] Govoni, F. et al., Science 364 (2019) 981.

[4] Nicastro, F. et al., Nature 558 (2018) 406.

[5] Ryu, D., Kang, H., and Biermann, P. L., Astron. Astrophys. 335 (1998) 19.

[6] Abreu, P. et al., Astropart. Phys. 35 (2012) 354.

[7] Aab, A. et al., EPJ C 75 (2015) 269.

[8] Bretz, H. P., PhD thesis, Rheinisch-Westphalian Technical University of Aachen, 2011.

[9] Lundquist, J. P. and Sokolsky, P. V., EPJ Web Conf. 210 (2019) 01006.

[10] Abbasi, R. U. et al., Astropart. Phys. 68 (2015) 27 .

[11] Abbasi, R. U. et al., Astrophys. J. 790 (2014) L21.

[12] Tinyakov, P. G. and Tkachev, I. I., Astropart. Phys. 24 (2005) 32.

[13] Kendall, M. G., Biometrika 33 (1945) 239.

[14] Abbasi, R. U. et al., Phys. Rev. Lett. 100 (2008) 101101.

[15] Ivanov, D., PhD thesis, Rutgers, the State University of New Jersey, 2012.

[16] Sagawa, H., in Proceedings, 33rd ICRC (ICRC2013): Rio de Janeiro, Brazil, July 2-9, page 0121, 2013. 